Noname manuscript No.

(will be inserted by the editor)

\title{
Voluntary contributions in a system with uncertain returns: A case of systemic risk
}

Annarita Colasante, Aurora Garcia-Gallego, Nikolaos

Georgantzis, Andrea Morone

\begin{abstract}
This paper investigates systemic risk that emerges from the interplay between uncertain returns to individual actions, uncertainty on others' behavior and all this filtered through individual attitudes towards risk. We design a finitely repeated linear public good experiment based on a voluntary contribution mechanism and analyze the effect of risky and uncertain returns on subjects' contributions. Results from a baseline treatment without uncertainty are compared with two risky treatments characterized by different values for the marginal per capita return. In the treatments with risk, subjects are randomly assigned to one out of three feasible marginal per capita returns, independently of what their individual contribution was. Results show that a sufficient level of uncertainty leads to significantly lower individual contributions. Furthermore, in a system with lower contributions due to uncertainty, subjects' risk aversion enhances the systemic risk, leading the system to collapse.
\end{abstract}

JEL: D84, E37, G12

Keywords: Uncertainty, systemic risk, exogenous risk, public good game, experiment. 


\section{Introduction}

Often, when we refer to an economic system, we consider a market situation with its demand and supply sides mostly inspired by the traditional view of non-cooperative interaction between sellers and buyers. In a recent paper, Barreda-Tarrazona et al. (2018) show that such a market system can also be viewed as a social dilemma in which socially better outcomes than the noncooperative equilibrium can be achieved if buyers and sellers behave in an altruistic way. For example, selling at a low price or producing enough to fully satisfy the demand can be seen as actions of altruism. More straightforward examples can be sought in the provision of public goods with resources obtained from the collection of taxes. In such a context, the literature on voluntary contributions to public goods becomes relevant also in the general context of economic systems.

In this paper we address the emergence of systemic risk in an economic system providing a public good which entails three different but interrelated phenomena: i. Uncertainty on the individual returns from the public good, ii. Strategic uncertainty due to 'others' behavior', and iii. Heterogeneity in the agents' attitudes towards risk. Imagine for example, a population of tax payers considering whether to evade or fully comply with their fiscal obligations. If in return to their taxes the state provides a health service of a random or fluctuating quality, depending, say, on the quality of hospitals or doctors available, the citizens' decisions regarding tax compliance may be negatively affected. At the same time, whether other citizens have complied or not with their obligations adds a further source of (strategic) uncertainty regarding the final quality of the health service received. Finally, both sources of uncertainty will be filtered through the tax-payers' risk attitudes and will, eventually, create the heterogeneously perceived systemic risk, which will lead to a variety of individual decisions and, thus, to a variety of potential final outcomes at an aggregate level. Our analysis illustrates the emergence of systemic risk in a rather broad family of cases in which the interplay between uncertain returns to individual actions and uncertainty on others' behavior is filtered through individual attitudes towards risk, leading not only to collectively worse outcomes but, in the extreme, even to the collapse of the system.

After the 2007-08 financial crisis, systemic risk has been part of a rapidly growing research agenda. As defined in Kaufman and Scott [2003], systemic risk refers to the probability of collapse of an entire system due to the correlation among most of the parts of the system itself. The majority of references in this field identify the interconnections among banks and institutions (Berardi and Tedeschi [2017], Acemoglu et al. [2015], Freixas et al. [2000] and Recchioni and Tedeschi [2017]) as the main systemic risk determinants. From a different perspective, Schwarcz [2008] provides an alternative definition for the same term: "systemic risk results from a type of tragedy of the commons in which market participants lack sufficient incentive, absent regulation, to limit risk-taking in order to reduce the systemic danger to others". Always in a financial environment, Masciandaro and Passarelli [2013] describe financial systemic risk as a pollution issue, as a financial risk externality. They point out that "systemic risk is a peculiar case of externality resulting from contagion effects", and that "any single financial portfolio produces a certain amount of systemic risk pollution, even an extremely small one". Therefore, since free-riding leads to excess risk production, systemic risk can be defined as a negative externality.

The main aspect of a public good provision is that everyone in the society benefits from the public investment, independently of whether the beneficiary contributed at all. As a matter of fact, the public investment generates returns that normally are heterogenously exploited and that are independent of the contribution level. In order to build up policies aimed at sustaining the provision of public goods, we investigate how uncertain and heterogeneous returns from public goods affect the decision of whether and how much to contribute to a common project. The returns from public investments are determined by two main factors. Namely, the total amount 
contributed (i.e. the taxes paid), and the allocation of those contributions. If a majority of citizens prefer not to invest in the public project, a collapse of the system may occur and, consequently, public services may not be provided. Related to exactly this aspect of tax undercontribution, several experimental investigations (Listokin and Schizer [2013] and Doerrenberg [2015]) have shown that the policy of introducing earmarking in public expenditure reduces the subjects' willingness to evade taxes. Strictly speaking, the earmarking increases the transparency in the allocation of public contributions and, therefore, it reduces the uncertainty of future returns. As a result, earmarking encourages subjects to contribute more to public investments and, therefore, reduces the risk of collapse of the system.

In the standard setting of a public good game (PGG, henceforth), players simultaneously decide how much to contribute to the public good and, as a result, they face the natural uncertainty due to uncertainty on others' behavior (Messick et al. [1988]) at the moment of deciding whether and how much to contribute. Furthermore, previous related research like Fischbacher and Gächter [2010] and Morone and Temerario [2018], remarks how each subject's preferences are characterized by a different willingness to cooperate. Those papers find subjects behaving as free-riders, subjects that are altruistic and subjects that act as conditional cooperators. Players in a PGG are part of a randomly determined group in which different kind of people are matched, and where each individual has no prior information about the preferences of others in the group. Moreover, players decide simultaneously about their individual contribution to the public good. In other words, players decide under imperfect and uncertain information. When players play in a repeated and partners set-up, uncertainty may be reduced since subjects try to learn about others' preferences and future behavior by observing the decisions taken in previous periods. Seminal works by Andreoni [1988] and Andreoni and Croson [2008], remark that the explanation behind the usual pattern of decaying contributions is a combination of learning to play the dominant strategy and strategic play by self interested players. ${ }^{1}$ Research conducted by Neugebauer et al. [2009] sheds light on these results. In their experiment, authors elicit beliefs about others' contributions and compare results from the 'Info' treatment -where the individual contribution of each member is shown at the end of each period-, and the 'NoInfo' treament in which subjects only receive feedback about the aggregate value of the public good. The decline in contribution is observed solely in the 'Info' treatment, causing also correlation between beliefs and individual investment to rise. This may be interpreted according to the conditional cooperation strategy.

More specific to strategies used in a PGG, Fischbacher et al. [2001] find that around 50\% of the subjects show conditional behavior such that the own contribution increases in the other group members' average contribution. ${ }^{2}$ Furthermore, when allowed, being a conditional cooperator may imply punishing members of the group that free-ride in the previous period (Fehr and Gächter [2002]). It is reasonable to assume that in a PGG with uncertainty the proportion of free-riders would increase, being this fact by itself a source of systemic risk. In fact, one's unwillingness to contribute propagates through the conditional cooperation strategy, likely ending up with the failure in the provision of the public goods, presumably leading to a collapse of the whole system.

We aim at carrying out the analysis of systemic risk under the same perspective of Schwarcz [2008], as a problem of failure in the provision of public goods due to the lack of incentives provided by the uncertain marginal per capita return (MPCR, henceforth). Indeed, when uncertainty increases due to the fact that subjects are uncertain about the marginal return of their contributions, they may prefer not to invest at all in the common project. Based on this reasoning, we draw our first research question:

\footnotetext{
1 See Chaudhuri [2011] for a detailed survey on cooperation in laboratory public goods experiments.

2 The authors use a variant of the strategy method: subjects are asked to indicate, for each average contribution level of the group members, how much they want to contribute to the public good.
} 
RQ1: The number of free-riders is expected to increase with uncertainty.

In order to test RQ1, we propose a public good experiment with uncertain individual returns. In our setting, subjects do not know their MPCR when deciding their contribution level in each period. Three possible realizations of the MPCR are considered in our experiment, each randomly allocated within the 3-member-group. Each MPCR realization is equally likely, which implies that ties are possible. In order to test whether different degrees of uncertainty affect subjects' contribution levels, we run three different treatments. First, a baseline (BL) in which the MPCR takes the same value for all subjects and this is common knowledge, therefore subjects are certain about their MPCR. Second, a treatment with high risk (HR) and, third, a low risk (LR) treatment. The main difference between the treatments with risk, HR and LR, is the variance between the MPCR values. This aspect of our design is explained in detail in Section 3.

In this environment with induced uncertainty, the contribution level is expected to be affected by the individuals' risk preferences. Budescu et al. [1990] as well as Sabater-Grande and Georgantzis [2002], find that risk aversion negatively affects cooperation. Based on this evidence we formulate our second research question:

RQ2: Subjects that are more risk averse contribute significantly less to the public good, and this effect is intensified with risky returns.

Our results partially confirm the conjecture that uncertain future returns have a significant negative effect on individual contributions. Moreover, this negative effect is stronger in the treatment with higher risk. In general terms, compared with the non risky treatment, we find that the uncertainty on the individual degrees of appropriation of the public good leads to lower levels of individual involvement in the social project. Furthermore, evidence is found of a source of systemic risk in this context that emerges from the interplay between the aforementioned uncertain returns and individual risk attitudes. Specifically, the individuals' risk aversion degree enhances the system to collapse due to lower individual contributions under return uncertainty.

The rest of the paper is structured as follows. In Section 2 we review the relevant experimental literature on PGG with uncertainty. Section 3 is dedicated to the experimental design and procedures. The theoretical framework and predictions are developed in Section 4. The analysis of our experimental data is detailed in Section 5. Section 6 concludes. Appendix A includes the instructions (translated from Spanish) given to the participants at the beginning of each session.

\section{Public good games with uncertainty}

Experimental research on linear PGG is extensive (Isaac et al. [1984]) and has been undertaken under different perspectives. Some of them are, for example, to introduce non-monetary incentives (Dugar [2013]), to allow for endogenous group formation (Brekke et al. [2011]), or to select the MPCR through a voting process (Colasante and Russo [2017]). Furthermore, cooperation in a public goods environment has been recently studied under uncertainty about the MPCR (BouluReshef et al. [2017]). More specifically, much interest has been put in studying the effect of risk in a public good environment, referring to situations in which agents are uncertain about the final returns but are aware of both the outcomes and their associated probabilities.

In this context, we will refer to uncertainty whenever the future outcomes are unknown for players. Furthermore, other sources of uncertainty have been incorporated in the standard game, for example, by introducing risky returns. In the context of a step-level public good, Au [2004] introduced risk on the provision threshold. In this setting, subjects were told that the threshold could take one of three different but equally likely values. The author obtained a lower level of 
contribution compared to that of a baseline treatment with a fixed threshold. Other example is the work by Wit and Wilke [1998] who investigated the effect of uncertainty on the provision point. In this setting, the threshold was randomly drawn from a uniform distribution -with the same mean of 500, and different range-, and the subjects were aware of the minimum and maximum values. In this study, a drop in contribution levels was also observed. In a related line of research, Milinski et al. [2008] analyzed collective risk dilemmas where the return depended on contributions exceeding a threshold. They addressed an interesting research question: "will a group of people reach a fixed target sum through successive monetary contributions, when they know they will lose all their remaining money with a certain probability if they fail to reach the target sum?" The authors found that, in case of high probability of losing all their remaining money (i.e. high probability of a serious natural disaster), half of the groups succeed in reaching the threshold, whereas the others only marginally failed. As a result, when the potential loss equals -or is lower- to the necessary average investment, the groups generally fail to reach the threshold. Individual behavior in low-probability-high-loss risk situations has been analyzed also by Ozdemir and Morone [2014], who found that subjects consider 'loss size' when making paying decisions.

Moving from the step-level to the standard linear PGG, an alternative way to increase uncertainty is to consider that the MPCR is risky. In an early paper, Dickinson [1998] compared the baseline treatment with a standard public good to one in which subjects knew the exogenous probability of getting the public good (named the 'uncertainty treatment'); an alternative treatment was considered in which the probability of getting the public good depended on the contribution at an aggregate level (named the 'incentive treatment'). In both treatments, the MPCR was homogeneous within the group. It is shown that playing under uncertain MPCR has a detrimental effect on contribution levels.

Following a similar line, several papers look at whether a risky MPCR negatively affects the willingness to contribute. Levati et al. [2009] test the impact of imperfect information on participants' contribution to the public good. In particular, they run two treatments, one with perfect information ( $P I$-treatment) and other with imperfect information about the MPCR $(I I-$ treatment), in the sense that the MPCR can take the values 0.4 or 1.1 with the same probability. Also, in their experiment there is a random extraction for each group, and therefore the value of the MPCR is heterogeneous between but not within groups. Results show that imperfect information entails lower average contributions and higher percentage of free-riding. However, in a follow-up experiment it has been shown that this effect may depend on parameterization (Levati and Morone [2013]). The authors assign different values to the MPCR, and compare the risk treatment with known probabilities with the uncertainty treatment where participants know just the two possible values of MPCR (either 0.6 or 0.9 ). In contrast with their findings in the previous study, contributions in both risky and uncertain treatments are not statistically different from contributions in the control treatment where a standard linear public good is implemented. Teyssier [2012] analyzes which type of intrinsic preferences drive an agent's behavior in a sequential PGG, depending on whether the agent is a first or second mover. Theoretical predictions are based on the heterogeneity of individuals in terms of social and risk preferences. Teyssier [2012] models preferences according to the inequity aversion model of Fehr and Schmidt [1999] and to the assumption of constant relative risk aversion. Risk aversion is significantly and negatively correlated with the contribution decisions of first movers. Second-movers with sufficiently high advantageous inequity aversion free-ride less and reciprocate more than others. Both results are predicted by her model. Nevertheless, no effect of disadvantageous inequity aversion of first-movers is found in the data, while theory predicted it. Her results underline the importance of taking into account the order of agents' play to correctly understand which preference influences cooperation in voluntary contribution mechanisms. 
So far, all references mentioned share the fact that returns are heterogenous only between -but not within- groups. In relation to this, Fischbacher et al. [2014] are the first to introduce heterogeneous returns within the group. In their experiment, there are a low and a high MPCR and subjects, split in groups of four, are aware that two per group will receive the high return and the other two the low one. Introducing this source of uncertainty is detrimental for contributions. By implementing a similar approach, Boulu-Reshef et al. [2017] run an experiment in which an individual MPCR value is drawn from a uniform distribution and, therefore, the MPCR is heterogeneous not only within the group but also between groups. The authors compare one treatment with certainty where the MPCR is homogeneous and known ex ante by subjects, with a treatment with complete uncertainty about the MPCR relative to the private consumption, and individually drawn after the contribution decision. They obtain that contributions are equivalent when the rate of return is predetermined and when it is uncertain, and show a similar decay over time. Therefore, in their setting uncertainty per se about the MPCR plays no role in the contribution decisions.

\section{Experiment}

We propose an experiment in which subjects play a finitely repeated version of a linear PGG. Our design aims at investigating the effect of risky and uncertain returns on individual contribution levels to a social project.

\subsection{Experimental design}

Three treatments are considered. In a baseline treatment (BL) subjects in groups of three play a standard PGG with a fixed and certain MPCR equal to $\alpha=0.6$. The other two treatments are risky: a high risk (HR) and a low risk (LR) treatment. Both risky treatments, HR and LR, differ only in the variance of the distributions from which the specific values of the MPCR are extracted.

Table 1: MPCR values, per treatment

\begin{tabular}{cccc}
\hline & BL & HR & LR \\
\hline $\bar{\alpha}$ & 0.6 & 0.9 & 0.75 \\
$\alpha$ & 0.6 & 0.6 & 0.6 \\
$\underline{\alpha}$ & 0.6 & 0.3 & 0.45 \\
\hline
\end{tabular}

Apart from the subjects' imperfect information about others' contributions, we introduce uncertainty about the value of MPCR. As already explained in the previous section, risky treatments in the literature have been usually characterized by the random selection between two values of the MPCR for each group. In our experiment, there are three different values of $\alpha$. Specifically, a high value $(\bar{\alpha})$, a medium value $(\alpha)$ and a low value $(\underline{\alpha})$. Table 1 shows the three values of the MPCR per treatment. These three values of $\alpha$ are the same for all groups within the same treatment and session. 
The values of the MPCR for treatments HR and LR have been chosen such that two conditions hold. On one hand, the average value $(\tilde{\alpha})$ must be equal to the MPCR in the baseline treatment (i.e., $\tilde{\alpha}=0.6$ ). On the other hand, the induced level of risk in HR must be higher than that in LR. To comply with the latter condition, the values of MPCR for treatment HR are such that the difference between the maximum and the minimum $\alpha$ is higher than the same difference for treatment LR. Formally, this means that $\left(\bar{\alpha}_{H R}-\underline{\alpha}_{H R}=0.6\right)>\left(\bar{\alpha}_{L R}-\underline{\alpha}_{L R}=0.3\right)$.

In every period, once contributions are made, for each subject within a group, the computer randomly selects, with replacement, a number from the set $X=\{1,2,3\}$. Once a single number has been assigned to each subject in each group, the computer ranks them. If the result is that each player is assigned a different number, the subject with number 1 in the group receives $\bar{\alpha}$, the subject with number 2 receives $\alpha$ and the one with number 3 receives $\underline{\alpha}$. Since the random extraction is made with replacement, we implicitly leave the possibility for a tie. In fact, it could be the case that every subject in the group gets either the highest or the lowest MPCR. ${ }^{3}$ In case of a tie, experimental instructions included the details on how ties would be solved during the session (see appendix A), so that subjects were informed before playing. In a session, the game was repeated over 10 rounds.

Returns in our set-up are risky, since participants know not only the values that the MPCR may take, but also the probability of getting one specific value for the MPCR. Subjects are also aware of the random allocation of the three possible values of the MPCR. Furthermore, returns are uncertain because the value of the MPCR is randomly assigned through an extraction process.

\subsection{Experimental procedures}

A total of 216 undergraduate students took part in the experiment. Each subject participated in just one session of one treatment. Hard copies of the instructions were distributed for each specific treatment (see Appendix A), and then the experimenter explained aloud the whole decision making process. A standard repeated linear PGG was played in treatment BL and the same game with risky returns was played in treatments HR and LR. Subjects were informed about the values of the MPCR $(\underline{\alpha}, \alpha, \bar{\alpha})$. In particular, participants in the BL treatment knew that the MPCR was the same for all subjects. Likewise, participants in treatments HR and LR knew that the MPCR was randomly assigned and, therefore, that it was independent of their contributions.

At the beginning of the session, each player received an endowment of $100 \mathrm{ECU}$, and had to decide -privately and anonymously- whether to keep the money or to contribute to a social project. By reading the instructions, subjects knew that they would play the same game for 10 rounds. Subjects' contribution decisions were taken before the disclosure of the values for MPCR. Participants were informed that a partner matching protocol was applied. During the session, subjects could see at the end of each round the individual contribution of each member of the group, the allocation of the MPCR among the members of their group, and own payoff for that period.

At the end of period 10, after a brief questionnaire about demographic data, we elicited individual risk aversion by implementing the lottery panels risk elicitation task of Sabater-Grande and Georgantzis [2002]. With this method, subjects were asked to choose their preferred lottery from four panels, knowing the probability to win the reported amounts. Table 2 reports the

\footnotetext{
3 The case in which all participants get the same benefit from the public good is not unrealistic. Consider, for instance, the public healthcare system. A person who receives medical care by a doctor with good skills do not exclude the possibility that other people may receive good care as well.
} 
summary of the lotteries of each panel of the test. Observe that the amount related to each probability increases from panel 1 (P1) to panel 4 (P4). Moreover, the higher the winning probability of the lottery chosen, the more risk averse the subject is. ${ }^{4}$ Risk neutral and risk loving subjects will choose the riskiest option available.

Table 2: The lottery panels risk elicitation task

\begin{tabular}{rcccccccccc}
\hline & \multicolumn{10}{c}{ Probability } \\
\hline Panels & 1 & 0.9 & 0.8 & 0.7 & 0.6 & 0.5 & 0.4 & 0.3 & 0.2 & 0.1 \\
\hline P1 & 1.00 & 1.10 & 1.30 & 1.50 & 1.70 & 2.10 & 2.70 & 3.60 & 5.40 & 10.90 \\
P2 & 1.00 & 1.20 & 1.50 & 1.90 & 2.30 & 3.00 & 4.00 & 5.70 & 9.00 & 19.00 \\
P3 & 1.00 & 1.70 & 2.50 & 3.60 & 5.00 & 7.00 & 10.00 & 15.00 & 25.00 & 55.00 \\
P4 & 1.00 & 2.20 & 3.80 & 5.70 & 8.30 & 12.00 & 17.50 & 26.70 & 45.00 & 100.00 \\
\hline
\end{tabular}

The experiment was programmed in z-Tree (Fischbacher [2007]) and conducted in the LEE experimental laboratory of the Universitat Jaume I (Castellón, Spain) in December 2016. We ran two sessions for each treatment with 36 participants per session, randomly distributed in groups of three. Because of the partners protocol, this yields 24 independent observations per treatment. Each session lasted around 30 minutes and the average earnings per subject were 16.75 Euro.

\section{Theoretical framework}

Consider the standard public good game. Subject $i$ 's payoff function is expressed as,

$$
\pi_{i}=e-c_{i}+\alpha \sum_{j=1}^{n} c_{j}
$$

where $e$ is the initial endowment, $c_{i}$ is subject $i$ 's individual contribution, and $\alpha$ is the MPCR. Parameter $\alpha<1$, implying that the subject payoff is decreasing with the individual contribution $\left(c_{i}\right)$, and increasing with the contribution of others $\left(c_{-i}\right)$.

In our framework, the MPCR is assigned in an individual way and without depending on the individual contribution. In each of the two risky treatments, three different values of $\alpha$ are considered. These values of $\alpha$ have been calibrated in order to reach a social dilemma situation. More specifically, the following conditions hold,

(i) $\underline{\alpha}<\alpha<\bar{\alpha}<1$

(ii) $\frac{1}{\tilde{\alpha}}>\frac{1}{N}$, where $\tilde{\alpha}=0.6$ is the average MPCR.

Condition (i) guarantees that the best individual strategy is to free-ride, while condition (ii) guarantees that, on average, the best strategy for the group -the Pareto optimum- is reached in case that every member of the group contributes with the whole endowment. In theory, condition (ii) holds in all treatments. In other words, given that subjects do not know ex-ante their own

\footnotetext{
4 For every lottery, the alternative outcome is a zero payoff. For a more detailed explanation of this test, see García-Gallego et al. [2012].
} 
MPCR, we reasonably assume that, when deciding, they take the average MPCR as a reference point.

Furthermore, the experimental treatments HR and LR are uncertain because subjects know just the probability with which they will get a MPCR for their investment, a value that may take one among three possible. The individual expected payoff is computed by considering every possible randomly assigned MPCR. It can be expressed as,

$$
E\left(\pi_{i}\right)=e-c_{i}+\left(\frac{8}{27} \bar{\alpha}+\frac{11}{27} \alpha+\frac{8}{27} \underline{\alpha}\right) \cdot \sum_{j=1}^{n} c_{j}
$$

which is equivalent to:

$$
E\left(\pi_{i}\right)=e-c_{i}+\alpha \cdot \sum_{j=1}^{n} c_{j}
$$

Therefore, the expected payoff is computed by taking the average MPCR into account. We use the linear net payoff function and simulate the best response function of subject $i$ with respect to the contribution of subject $j$. Observe in Figure 1 that, as subject $i$ 's contribution increases and subject $j$ 's contribution decreases, subject $i$ gets a negative net payoff. Moreover, according to the calibration of the MPCR values in Table 1, it is straightforward that the best individual strategy is to free-ride. In other words, for every value of $\alpha$, subject $i$ gets the maximum net payoff by no contributing at all $\left(c_{i}^{*}=0\right)$.

\section{Results}

In the next two subsections we present the analysis of our experimental data. First, we show the descriptive statistics of the distribution of contribution levels. Second, we elaborate an econometric modelization.

\subsection{Descriptive analysis}

A central goal of our study is to test whether the existence of risky returns in the system is detrimental for cooperation, in the sense of contributing less to the common project. We report in Figure 2 the average contribution per period and per treatment. This figure shows that: $i$ ) the average contribution follows the same non-linear pattern in all treatments; $i i$ ) the contribution level in the first period is significantly different from the one in the late periods in all treatments; iii) in comparison with the other two treatments, treatment HR shows the lowest average contribution; $i v$ ) a doomsday effect is clear in all treatments.

Tests $t$ and Mann Whitney have been calculated in order to compare, respectively, the mean and median contributions in treatments HR and LR with respect to contribution levels in the baseline BL. Table 3 shows, on the left-hand side, the descriptive statistics of the contribution distribution and, on the right-hand side, the values for the aforementioned tests. Observe that only in treatment HR individual contributions are significantly different from those in BL, something that is intuitively confirmed also graphically. In treatment LR, contributions are slightly lower than in the BL, but the difference is not statistically significant. 


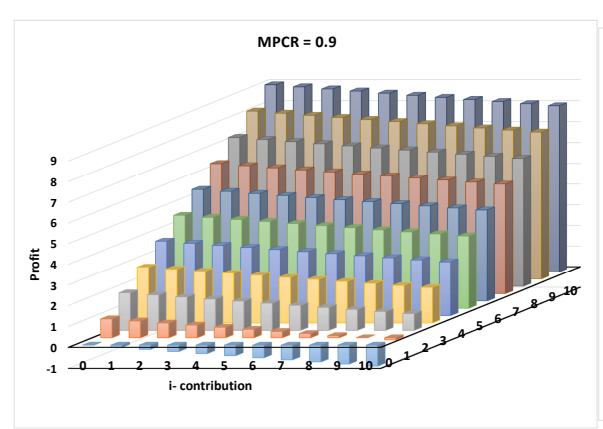

(a)

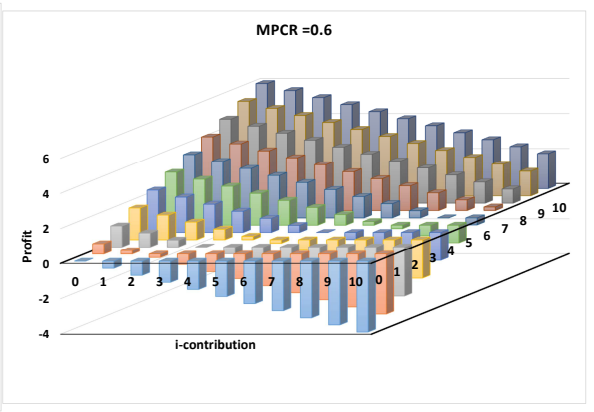

(b)

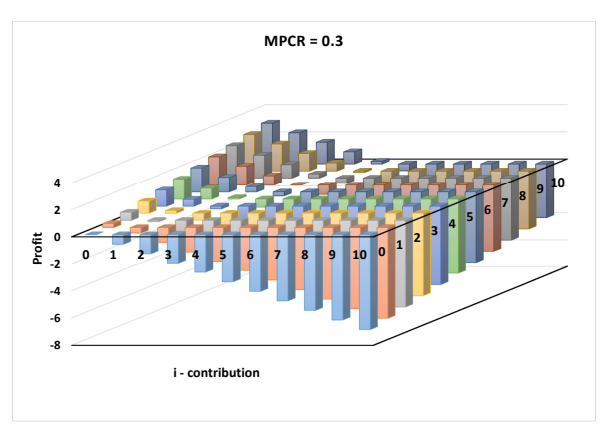

(c)

Fig. 1: Simulated individual net payoff. The interval of contribution levels $c \in(0,100)$ has been rescaled with the interval $c \in(0,10)$ aiming at having better quality pictures. Each bar represents the net profit of subject $i$ depending on own as well as subject $j$ 's contribution.

Table 3: Descriptive statistics of the contribution levels, per treatment

\begin{tabular}{lcccc|ll}
\hline Treatment & $\mathrm{N}$ & Mean & Median & $\mathrm{Sd}$ & $\mathrm{t}$-test & Mann Whitney test \\
\hline BL & 72 & 36.87 & 30 & 31.29 & - & - \\
$\mathrm{HR}$ & 72 & 29.80 & 20 & 28.95 & $\mathrm{t}=4.55^{* * *}$ & $\mathrm{z}=-3.83^{* * *}$ \\
LR & 72 & 35.31 & 25 & 32.51 & $\mathrm{t}=0.93$ & $\mathrm{z}=-0.79$ \\
\hline$* * *$ & & &
\end{tabular}

We have an interpretation with respect to the, common to all treatments, non-linear shape of the average contribution distribution. Our intuition is that subjects may be playing conditional cooperation, in the sense that an increase in the group contribution in one round makes the subject prone to contribute more to the public project in the next round. Furthermore, playing conditional cooperation means that when the subject detects that not everyone within the group is contributing at his/her same level, the incentive to reduce the own contribution increases in the next round. We speculate this is the kind of behavior behind the significant drop in average contribution after the "peak". Moreover, although the average contribution has a similar shape in all treatments, the difference in the timing observed in treatment HR may indicate that subjects in this treatment trust less and need more time to significantly increase their contribution.

Observe in Figure 2 how different the first period contribution levels are among treatments. The difference, although apparently big, is not statistically significant. However, the share of 


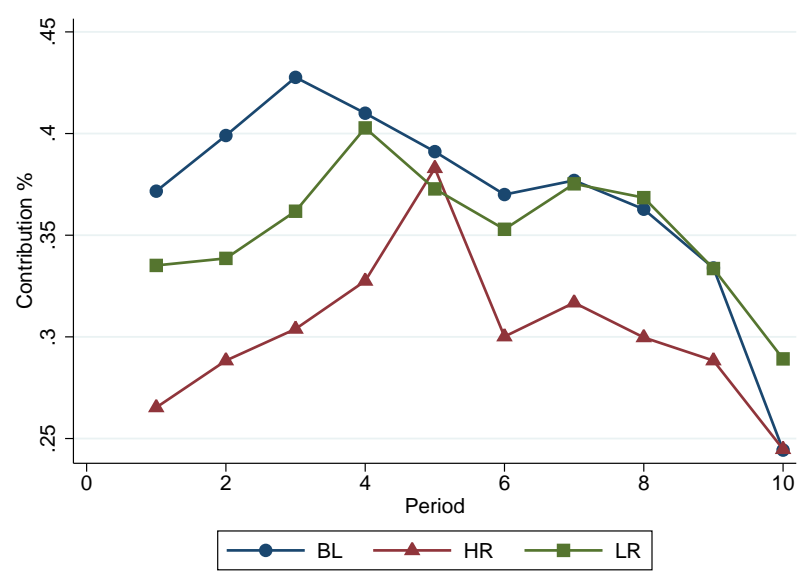

Fig. 2: Average group contribution over time in each treatment.

subjects contributing the whole endowment (i.e., $\mathrm{c}_{i}=100$ ) in the first period is equal to $8 \%$ in the baseline BL, $1 \%$ in HR, and $8 \%$ in LR. Accordingly, it seems that when subjects play the first round, they are not able to perceive any difference between a common MPCR (treatment $\mathrm{BL}$ ) for all players and receiving one of the $\alpha$ possible in treatment LR.

Although the general effect highlights an inverse relationship between risk and willingness to contribute to the public good, we observe no significant differences in LR with respect to the baseline BL. Consequently, we observe that only under sufficiently high risk the contribution levels can be significantly reduced. Analyzing more deeply this result in order to test our research question RQ1, we check whether a higher level of risk implies an increase in the number of free-riders. For each treatment, we compute the share of free-riders (FR), i.e. subjects whose contribution is zero in period $t$ (i.e., $c_{i t}=0$ ) and full cooperators $(\mathrm{FC})$, the ones that invest the whole endowment in the public good in period $t$ (i.e., $c_{i t}=100$ ).

In Figure 3 we show the percentage of FR and FC subjects per treatment. The share of freeriders per treatment is of $19 \%(\mathrm{BL}), 15 \%(\mathrm{HR})$ and $10 \%(\mathrm{LR})$, which corresponds to 14,11 and 7 subjects, respectively. Despite the fact that the average contribution is the highest in BL, the highest rate of free-riders is unexpectedly found also in the baseline. One could argue that this anomalous result may possibly be due to the presence of a sufficient number of full cooperators in our sample. However, if we look at the percentage of $\mathrm{FC}$ per treatment, we find that the lowest share $(6 \%)$ corresponds to treatment BL. Thus, the descriptive analysis of our data so far gives us evidence that more risky returns do not necessarily imply a higher probability of free-riding. Consequently, our first research question is not confirmed.

Going further in our analysis, we investigate the difference in contribution levels by splitting the subject pool according to a simple criterion. Specifically, the distribution of individual contribution shown in Figure 4 highlights that in the BL treatment a majority of subjects contribute either zero or a positive amount close to $60 \mathrm{ECU}$. On the other hand, in treatments HR and LR the majority of subjects invest an amount close to 20 ECU. Using this evidence, we exogenously choose a fix threshold equal to $50 \mathrm{ECU}$ and split our subjects sample in two groups: 'cooperators' (C), if contributing more than $50 \%$ of their endowment (i.e., 50 ECU); and 'no-cooperators' (NC) otherwise. This classification allows us to detect whether the highest contribution in treatment BL depends on the amount of free-riders or, alternatively, on the amount of full cooperators in the system. By observing Figure 5, it is quite straightforward to see that the highest share of 


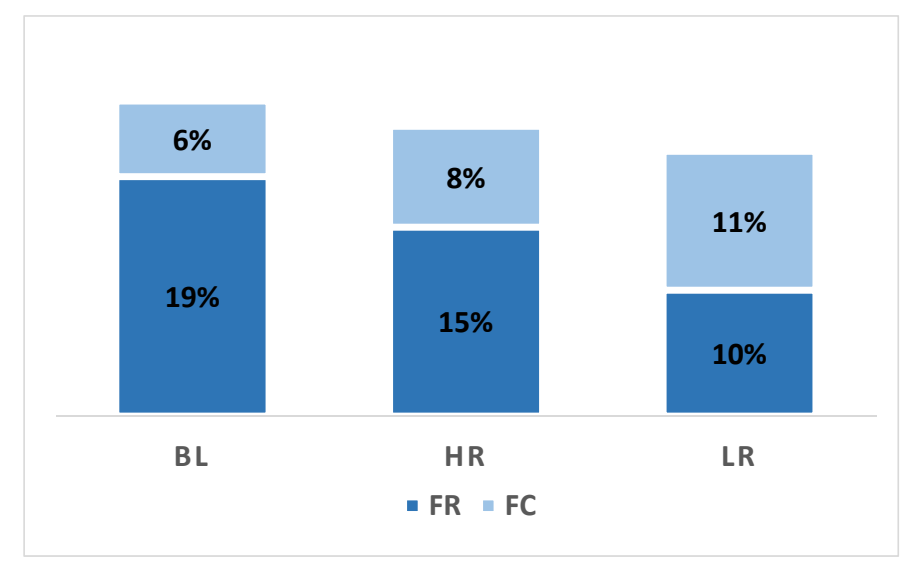

Fig. 3: Percentage of free-riders (FR) and full cooperators (FC), per treatment.

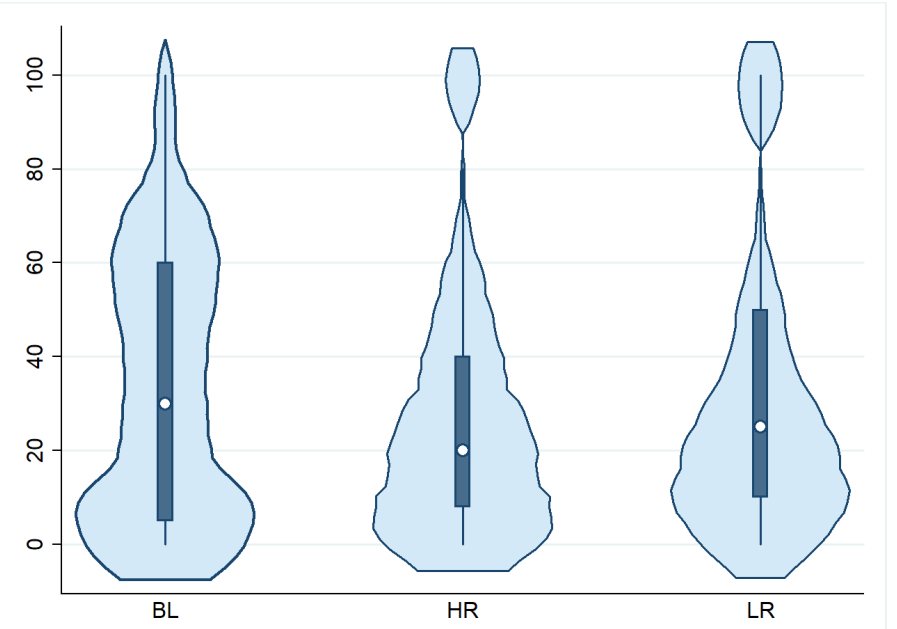

Fig. 4: Violin plot of the individual contribution in each treatment (the white dots indicate median values).

subjects willing to contribute more than $50 \%$ of their endowment corresponds to treatment BL, while the lowest share is that of treatment HR. Therefore, being uncertain about the MPCR makes the subject's returns more volatile and, consequently, the best strategy is to contribute a low positive amount to the public good. With such a strategy, subjects may reduce the risk related to the MPCR, which can be determined through two main factors. On the one hand, subjects know that the probability to get either the highest or the lowest MPCR is independent of their contribution, being this an incentive to contribute zero or a very low amount. On the other hand, subjects decide not to free-ride motivated by some other regarding preferences like, for example, reciprocity or fairness. In other words, subjects are aware of the fact that their individual contribution will be common knowledge at the end of each period and that, as a result, a subject with zero contribution may be the starting point of a domino effect that would end up in a system collapsing with each member of the group contributing zero. 


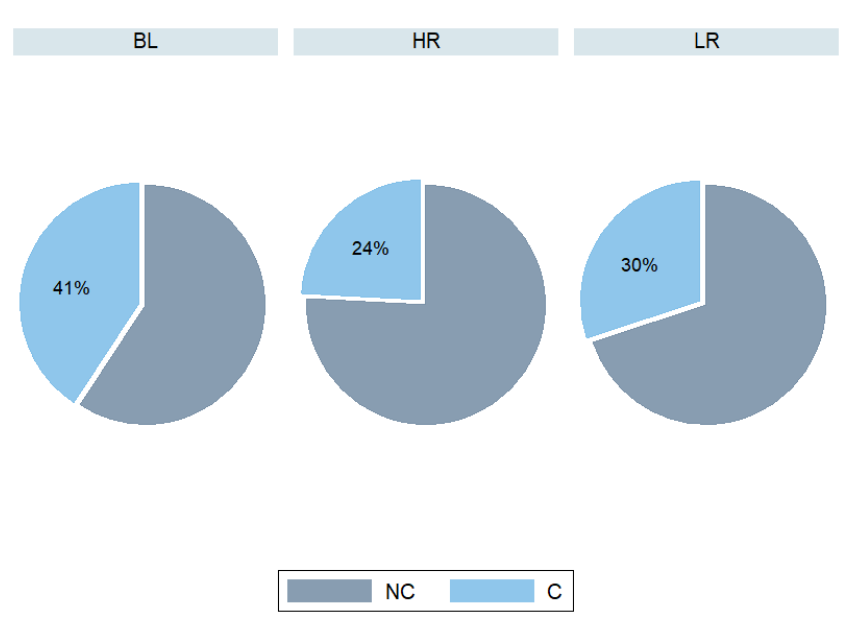

Fig. 5: Distribution of cooperators with $c \geq 50 \%$ (C) and no cooperators with $c<50 \%$ (NC), by treatment.

Let us summarize our findings so far. The existence of risky MPCR in the system leads to a decline in contributions which is statistically significant only in treatment HR, where the variance in the distribution of the values MPCR is the highest among all treatments. Furthermore, no evidence is found supporting research question RQ1, in the sense that risky returns do not necessarily cause an increase in free-riding. Interestingly, we find that induced uncertainty reduces the general willingness to contribute. Thus, low contribution levels remaining stable over time are observed, being more intense in treatment HR. This result may be evidence of subjects willing to contribute but at a low rate, likely caused by the high level of uncertainty on future returns.

\subsection{Econometric analysis}

In order to test our research questions, we estimate the effect of two different sets of variables on the probability to be a cooperator. This means that our dependent variable is the probability of being a cooperator, thus contributing more than half of the endowment (50 ECU in our design). Specification (1) in Table 4 is helpful to understand the impact of uncertainty on individual contribution. Therefore, such specification only includes the following explanatory variables: two treatment dummies, one for HR and one for LR; subject $i$ 's MPCR for the previous period $\left(\alpha_{i t-1}\right)$, and the MPCR for subject $j$ of the same group ${ }^{5}\left(\alpha_{j t-1}\right)$; finally, we include variables Factor 1 and Factor 2, two additional measures for individual risk aversion. These measures are computed according to the evidence shown in García-Gallego et al. [2012]. In particular, Factor 1 is equal to the average choice across the four panels presented in Table 2, being therefore a straight measure of risk aversion. In particular, the higher the value of Factor 1 the more risk averse the subject is. With respect to Factor 2, it measures the sensitivity to variations in the return to risk. Therefore, Factor 2 is a measure of the tendency of subjects towards varying their choices as they move from panel 1 to panel 4 . Specifically, it is computed as $[2(P 1-P 4)]+(P 2-P 3)$. Notice that, as shown in Table 4, we consider two models in order to isolate the effect due to risky MPCR from the effect of individual risk aversion.

\footnotetext{
${ }^{5}$ We must not include the MPCR of all members of the group, since this would result in a collinearity problem.
} 
Table 4: Probit panel regression with random effects. Dependent variable: probability to contribute more than $50 \%$ of the endowment

\begin{tabular}{|c|c|c|}
\hline & \multicolumn{2}{|c|}{ Specification (1) } \\
\hline & $\begin{array}{c}\text { Model (1) } \\
\operatorname{Pr}\left(C=1 \mid X_{i t}\right)\end{array}$ & $\begin{array}{c}\text { Model (2) } \\
\operatorname{Pr}\left(C=1 \mid X_{i t}\right)\end{array}$ \\
\hline $\mathrm{HR}$ & $\begin{array}{c}-0.95^{* * *} \\
(0.348)\end{array}$ & $\begin{array}{c}-0.93^{* * *} \\
(0.347)\end{array}$ \\
\hline LR & $\begin{array}{l}-0.63^{*} \\
(0.347)\end{array}$ & $\begin{array}{l}-0.59^{*} \\
(0.347)\end{array}$ \\
\hline$\alpha_{t-1}$ & $\begin{array}{c}-0.44 \\
(0.395)\end{array}$ & $\begin{array}{c}-0.44 \\
(0.396)\end{array}$ \\
\hline$\alpha_{t-1, j \neq i}$ & $\begin{array}{l}-0.74^{*} \\
(0.402)\end{array}$ & $\begin{array}{l}-0.77^{*} \\
(0.403)\end{array}$ \\
\hline Factor 1 & & $\begin{array}{c}-0.49 \\
(0.411)\end{array}$ \\
\hline Factor2 & & $\begin{array}{c}0.22 \\
(0.175)\end{array}$ \\
\hline Constant & $\begin{array}{c}0.24 \\
(0.476)\end{array}$ & $\begin{array}{c}0.45 \\
(0.532)\end{array}$ \\
\hline Observations & 1944 & 1944 \\
\hline Number of subjects & 216 & 216 \\
\hline Wald $\chi^{2}$ & $11.16^{* *}$ & $14.40^{* *}$ \\
\hline LR test $\chi^{2}$ & $848.22^{* * *}$ & $841.36^{* * *}$ \\
\hline
\end{tabular}

Standard errors in parentheses

Results from the regressions reinforce our previous findings. Specifically, the MPCR does not affect the actual decision of the subject of contributing to the public good with more than half of the endowment. Interestingly, however, the MPCR assigned to other subjects in the group in the previous period significantly reduces the probability of behaving as a cooperator in the present period. One may speculate that, even if subjects are aware that the MPCR is randomly assigned, their willingness to cooperate is negatively affected -a sort of envy- by the higher potential earnings of their partners. Results from Model (2) in this table shows that risk aversion plays no role in determining the probability to be a cooperator, while participating in one of the two risky treatments reduces such probability.

Specificacion (2) inTable 5 includes two models that allow us to test our second research question and, additionaly, our conjecture about the conditional cooperator behavior. In other words, with models (3) and (4) we aim at measuring the impact of the level of risk aversion and of others' contribution on the probability to be a cooperator. More specifically, we test the effect of: $(i)$ other members' contribution, and $(i i)$ the interaction between risk aversion and uncertainty. In particular, this specification includes the following explanatory variables: the aggregate contribution of the other members of the group in the previous period (Other $s^{\prime}$ contr $\left._{t-1}\right)$; the interaction between others' MPCR and their contribution; the measures of risk aversion interacted either with variable ${ }^{6} r$-in model (3)- or with the treatment dummies in model (4).

Observe Table 5. It is quite straightforward to see that subjects behave as conditional cooperators. In other words, the higher the others' contribution in the previous period, the higher the probability to be a cooperator in this round. The interaction between others' contribution and returns helps us to measure the existence of any correlation between these two variables. The estimation of the model reveals that the interaction is no significant, implying that subjects are

\footnotetext{
6 Variable $r$ is equal to the difference between $\bar{\alpha}$ and $\alpha$. This variable takes the values of $0,0.3$ and 0.15 for treatments $\mathrm{BL}, \mathrm{HR}$ and $\mathrm{LR}$, respectively.
} 
Table 5: Probit panel regression with random effects and variable interactions. Dependent variable: probability to contribute more than $50 \%$ of the endowment

\begin{tabular}{|c|c|c|}
\hline & \multicolumn{2}{|c|}{ Specification (2) } \\
\hline & $\begin{array}{c}\text { Model (3) } \\
\operatorname{Pr}\left(C=1 \mid X_{i t}\right)\end{array}$ & $\begin{array}{c}\text { Model (4) } \\
\operatorname{Pr}\left(C=1 \mid X_{i t}\right)\end{array}$ \\
\hline Others' $\operatorname{contr}_{t-1}$ & $0.02^{* * *}$ & $0.02 * * *$ \\
\hline \multirow{2}{*}{$(\alpha * c)_{t-1, j \neq i}$} & $\begin{array}{l}(0.004) \\
-0.02\end{array}$ & $\begin{array}{l}(0.004) \\
-0.01\end{array}$ \\
\hline & $(0.007)$ & $(0.007)$ \\
\hline \multirow{2}{*}{$(\alpha * c)_{t-1, k \neq j}$} & -0.01 & -0.01 \\
\hline & $(0.007)$ & $(0.007)$ \\
\hline \multirow[t]{2}{*}{ Factor1 } & 0.22 & 0.29 \\
\hline & $(0.505)$ & $(0.511)$ \\
\hline \multirow[t]{2}{*}{ Factor2 } & 0.26 & 0.27 \\
\hline & $(0.214)$ & $(0.226)$ \\
\hline \multirow[t]{2}{*}{ Factor $1^{*} \mathrm{r}$} & -2.42 & \\
\hline & $(1.537)$ & \\
\hline \multirow[t]{2}{*}{ Factor $2^{*} \mathrm{r}$} & -0.36 & \\
\hline & $(1.057)$ & \\
\hline \multirow[t]{2}{*}{ Factor $1 * \mathrm{HR}$} & & $-0.75^{*}$ \\
\hline & & $(0.410)$ \\
\hline \multirow[t]{2}{*}{ Factor $1 *$ LR } & & -0.71 \\
\hline & & $(0.499)$ \\
\hline \multirow[t]{2}{*}{ Factor $2 * H R$} & & -0.10 \\
\hline & & $(0.316)$ \\
\hline \multirow[t]{2}{*}{ Factor $2 *$ LR } & & -0.12 \\
\hline & & $(0.407)$ \\
\hline \multirow[t]{2}{*}{ Constant } & $-2.13^{* * *}$ & $-2.10 * * *$ \\
\hline & $(0.258)$ & $(0.263)$ \\
\hline Observations & 1944 & 1944 \\
\hline Number of subjects & 216 & 216 \\
\hline Wald $\chi^{2}$ & $168.49^{* *}$ & $169.43^{* * *}$ \\
\hline LR test $\chi^{2}$ & $424.75^{* * *}$ & $420.91^{* * *}$ \\
\hline
\end{tabular}

aware of the randomness in the MPCR assignment process. Furthermore, even though decisions are made in a risky environment, the model shows that individual risk aversion plays no role in the probability of behaving as a cooperator. By focusing on results of model (4), it is interesting to observe that the interaction between the degree of risk aversion and the dummy for treatment HR is negative and statistically significant. This means that it is less likely that risk averse subjects contribute with more than half of their endowment whenever a high degree of risk is introduced in the system. Potentially, a high uncertainty about future returns constitutes an important source of systemic risk. In other words, in order to face uncertainty, risk averse subjects will less likely behave as cooperators. This effect, together with the effect from others' contribution, will potentially result in others in the group imitating this behavior, consequently resulting in a failure of the system, ending with the no provision of the public good.

Finally, we explore the dynamics of the session, checking whether the features of our experimental design affect the main results in terms of both conditional cooperation and risk aversion. On the one hand, in our experimental design we have implemented a partner matching protocol so that the composition of groups remains constant along the session. On the other hand, at the end of each period we have provided information about contribution of others in the group. We present in Table 6 a dynamic linear panel regression that measures the impact of lagged own 
contribution $\left(\mathrm{c}_{i t-1}, \mathrm{c}_{i t-2}\right)$, lagged others' contribution (Others' contr $_{t-1}$ ) and risk aversion levels on individual contribution. Results are in line with those from the probit regressions shown in Tables (4) and (5). Additionally, we observe a path depedence in the individual contribution, since past values strongly affect the current level of contribution. Hence, we may conclude that our results in terms of conditional cooperation and of risk aversion are not significantly affected by the partner matching protocol.

Table 6: Dynamic panel regression. Dependent variable: individual contribution. Instruments: lagged values of individual contribution, others' contribution and MPCRs. Sargan test of overidentifying restriction: $\chi^{2}=$ $106.41(0.31)$

\begin{tabular}{lcc}
\hline & & $\mathrm{c}_{t}$ \\
\hline & $\mathrm{c}_{i t-1}$ & $0.16^{* * *}$ \\
& & $(0.02)$ \\
& $\mathrm{c}_{i t-2}$ & $0.10^{* * *}$ \\
& & $(0.01)$ \\
& & $0.13^{* * *}$ \\
& & $(0.02)$ \\
& Factor1 & -2.91 \\
& & $(5.02)$ \\
& Factor2 & 4.81 \\
& & $(3.43)$ \\
\hline Observations & & \\
Number of subjects & & 1512 \\
Wald $\chi^{2}$ & & 216 \\
AR $(1)$ & & \\
AR $(2)$ & & \\
\hline$* * * \mathrm{p}<0.01, * * \mathrm{p}<0.05,{ }^{*} \mathrm{p}<0.1$ & $-0.38(0.71)$ \\
Standard errors in parentheses &
\end{tabular}

Summing up our results, we have found that introducing an additional source of uncertainty results in a decrease in contribution levels but it does not necessarily increase the number of free-riders. Such a cutback is due to a possible strategy carried out by subjects in order to reduce the risk of getting the lowest MPCR, for example by contributing each period a low but positive amount. Furthermore, not to act as a free-rider has pushed the subjects to behave as conditional cooperators. Our research question RQ1 is ultimately rejected. Furthermore, the individual risk aversion level does not significantly affect the subject contribution decision. Only if we consider the interaction with the treatment variable, we find out that the reluctance to cooperate by risk averse subjects emerges in the high risk environment. In summary, not enough evidence is found to reject $\mathrm{RQ2}$.

\section{Conclusions}

A system's risk may originate from uncertainty due to external and institutional factors, uncertainty due to interaction between individuals and due to the way in which these risks are filtered through the attitudes of people towards uncertainty. This paper reports results from an experiment in which individual attitudes towards risk are accounted for in a set-up in which the returns from interacting agents' behavior in the context of a PGG are also uncertain. Specifically, we aim at testing two research questions. On one hand, we expect a larger share of free-riding 
behavior. On the other hand, we speculate that more risk averse subjects will contribute less to the public good, being this effect intensified by the risky returns.

We have designed a public good experiment with uncertain individual returns, that is, subjects, when deciding their contribution level each period, ignore their MPCR. We have considered three treatments, each corresponding to three possible realizations of the MPCR, randomly allocated for each of the 3-member-group. Subjects play a finitely repeated version of a linear PGG. In the baseline treatment (BL) subjects in groups of three play a standard PGG with a fixed and certain MPCR. The other two treatments correspond to heterogeneous distributions of the MPCR, one with higher variance than the other.

Our results partially confirm the conjecture that uncertain future returns have a significant negative effect on individual contributions but it does not imply an increase of free-riders. On the contrary, significant evidence has been found of subjects behaving as conditional cooperators, contributing more the more the others in the group contribute. The negative effect of uncertainty in the contribution levels has showed stronger in the treatment with higher risk. In general terms, compared with the baseline, uncertain returns lead to lower levels of individual investment in the social project. Furthermore, the individual risk aversion level does not significantly affect the subject contribution decision. Only if we consider the interaction with the treatment variable, we find out that the reluctance to cooperate by risk averse subjects emerges in this uncertain environment. Consequently, the hypothesis that more risk aversion implies lower levels of contribution can not be rejected.

The existence of risky MPCR in the system leads to a decline in contributions which is statistically significant only in treatment HR, where the difference between the minimum and the maximum value of MPCR is large enough. Furthermore, no evidence is found supporting research question RQ1, that is, risky returns do not cause an increase in the share of free-riders. Interestingly, we find that induced uncertainty reduces the general willingness to contribute and, consequently, we observe low levels of contribution that remain stable over time, especially in HR. This result may be an evidence of subjects willingness to contribute but at low rate, likely caused by the high level of uncertainty on future returns.

More specifically, the individuals' degree of risk aversion enhances lower individual contributions under return uncertainty, thus making the system to collapse in the middle run. A relevant policy implication of our analysis is that more egalitarian systems would suffer less from the effect that individual risk aversion might have on the reduction of individual contributions, occasionating the potential collapse of the social/economic system. 


\section{References}

Acemoglu, Daron, Asuman Ozdaglar, and Alireza Tahbaz-Salehi (2015) Systemic risk and stability in financial networks. American Economic Review 105.2: 564-608.

Andreoni, James (1988) Why free ride? Strategies and learning in public good experiments. Journal of Public Economics 37: 291-304.

Andreoni, James, and Rachel Croson (2008) Partners versus strangers: random rematching in public goods experiments. In C. PLott and V.L. Smith (Eds.), Handbook of experimental economics results pp. 776-783, Amsterdam: North-Holland.

Au Winton (2004) Criticality and environmental uncertainty in step-level public goods dilemmas. Group Dynamics: Theory, Research, and Practice 8.1:40-61.

Barreda-Tarrazona, Iván, Aurora García-Gallego, Nikolaos Georgantzis, and Nicholas Ziros (2018) Market games as social dilemmas. Journal of Economic Behaviour and Organization 155:435-444.

Berardi, Simone, and Gabriele Tedeschi (2017) From banks' strategies to financial (in)stability. International Review of Economics and Finance 47: 255-272.

Boulu-Reshef, Beatrice, Samuel H. Brott, and Adam Zylbersztejn (2017) Does uncertainty deter provision of public goods?. Revue Économique 5:16-23.

Brekke, Kjell Arne, Karen Evelyn Hauge, Linda Jo Thori, and Karine Nyborg (2011) Playing with the good guys. A public good game with endogenous group formation. Journal of Public Economics 95.9-10:1111-1118.

Budescu David V., Amnon Rapoport, and Ramzi Suleiman (1990) Resource dilemmas with environmental uncertainty and asymmetric players. European Journal of Social Psychology 20.6:475-487.

Chaudhuri, Ananish (2011) Sustaining cooperation in laboratory public goods experiments: a selective survey of the literature. Experimental Economics 14:47-83.

Colasante, Annarita, and Alberto Russo (2017) Voting for the distribution rule in a public good game with heterogeneous endowments. Journal of Economic Interaction and Coordination 12.3:443-467.

Dickinson, David L (1998) The voluntary contributions mechanism with uncertain group payoffs. Journal of Economic Behavior and Organization 35.4:517-533.

Doerrenberg, Philipp (2015) Does the use of tax revenue matter for tax compliance behavior? Economics Letters 128:30-34.

Dugar, Subhasish (2013) Non-monetary incentives and opportunistic behavior: Evidence from a laboratory public good game. Economic Inquiry 51.2:1374-1388.

Fehr, Ernst, and Simon Gächter (2002) Altruistic punishment in humans. Nature 415.6868:137.

Fehr, Ernst, and Klaus M. Schmidt (1999) A theory of fairness, competition, and cooperation. The Quarterly Journal of Economics 114.3:817-868.

Fischbacher, Urs, Simon Gächter, and Ernst Fehr (2001) Are people conditionally cooperative? Evidence from a public goods experiment. Economics Letters 71.3:397-404.

Fischbacher Urs (2007) z-Tree: Zurich toolbox for ready-made economic experiments. Experimental Economics 10.2:171-178.

Fischbacher Urs, and Simon Gächter (2010) Social preferences, beliefs, and the dynamics of free riding in public goods experiments. American Economic Review 100.1:541-556.

Fischbacher, Urs, Simeon Schudy, and Sabrina Teyssier (2014) Heterogeneous reactions to heterogeneity in returns from public goods. Social Choice and Welfare 43.1:195-217.

Freixas, Xavier, Bruno M. Parigi, and Jean-Charles Rochet (2000) Systemic risk, interbank relations, and liquidity provision by the central bank. Journal of Money, Credit and Banking $32(3): 611-638$ 
García-Gallego, Aurora, Nikolaos Georgantzís, Ainhoa Jaramillo-Gutiérrez, and Melanie Parravano (2012) The lottery-panel task for bi-dimensional parameter-free elicitation of risk attitudes. Revista Internacional de Sociología 70.1:53-72.

Isaac, R. Mark, James M. Walker, and Susan H. Thomas (1984) Divergent evidence on free riding: An experimental examination of possible explanations. Public Choice 43.2:113-149.

Kaufman, George G., and Kenneth E. Scott (2003) What is systemic risk, and do bank regulators retard or contribute to it?. The Independent Review 7.3:371-391.

Levati, M. Vittoria, Andrea Morone, and Annamaria Fiore (2009) Voluntary contributions with imperfect information: An experimental study. Public Choice 138.1:199-216.

Levati, M. Vittoria, and Andrea Morone (2013) Voluntary contributions with risky and uncertain marginal returns: The importance of the parameter values. Journal of Public Economic Theory 15.5:736-744.

Listokin Yair, and David M. Schizer (2013) I like to pay taxes: Taxpayer support for governemt spending and the efficiency of the tax system. Tax Law Review 66:179.

Masciandaro, Donato, and Francesco Passarelli (2013) Financial systemic risk: Taxation or regulation?. Journal of Banking \& Finance 37.2: 587-596.

Messick, David M., Scott T. Allison, and Charles D. Samuelson (1988) Framing and communication effects on group members' responses to environmental and social uncertainty. Applied Behavioral Economics 2:677-700.

Milinski Manfred, Ralf D. Sommerfeld, Hans-Jürgen Krambech, Floyd A. Reed, and Jochem Marotzke (2008) The collective-risk social dilemma and the prevention of simulated dangerous climate change. Proceedings of the National Academy of Sciences 105.7:2291-2294.

Morone, Andrea, and Tiziana Temerario (2018) Is dyads' behaviour conditionally cooperative? Evidence from a public goods experiment. Journal of Behavioural and Experimental Economics 73:76-85.

Neugebauer, Tibor, Javier Perote, Ulrich Schmidt, and Malte Loos (2009) Selfish-biased conditional cooperation: On the decline of contributions in repeated public goods experiments. Journal of Economic Psychology 30.1:52-60.

Ozdemir, Ozlem, and Andrea Morone (2014) An experimental investigation of insurance decisions in low probability and high loss risk situations. Journal of Economic Interaction and Coordination 9.1:53-67.

Recchioni, M. Cristina, and Gabriele Tedeschi (2017) From bond yield to macroeconomic instability: A parsimonious affine model. European Journal of Operational Research 262.3: 1116-1135.

Sabater-Grande, Gerardo, and Nikolaos Georgantzis (2002) Accounting for risk aversion in repeated prisoners' dilemma games: An experimental test. Journal of Economic Behavior and Organization 48.1:37-50.

Schwarcz, Steven L. (2008) Systemic risk. Georgetown Law Journal 97: 193-249.

Teyssier, Sabrina (2012) Inequity and risk aversion in sequential public good games. Public Choice 151.1: 91-119.

Wit, Arjaan, and Henk Wilke (1998) Public good provision under environmental and social uncertainty. European Journal of Social Psychology 28.2:249-256. 


\section{A Instructions to subjects (translated from Spanish)}

Thank you very much for being here. The instructions are identical to all participants. Read them carefully. If you have any questions or concerns, please raise your hand and we will answer your questions individually. During the session, it is strictly forbidden to communicate with the other participants. The unit of experimental money will be the ECU (Experimental Currency Unit), where $100 \mathrm{ECU}=10$ Euro. At the end of the session one of your decisions will be randomly chosen. Note that all choices are equally likely. The experimental payoff corresponding to the selected decision will be calculated, converted to Euro, and paid to you (privately) in cash.

The Experiment (Baseline (BL) treatment)

The experiment consists of 10 independent periods, in which you will interact with 2 other participants in the session. The 3 of you form a group that will remain THE SAME in all periods. The identity of the participants of your group will not be revealed to you at all during the whole session. At the beginning of each period, each participant receives an endowment of $100 \mathrm{ECU}$. In any period, each member of the group has a decision to make.

Every period, you have to decide how much of your endowment you want to contribute to a common project. Your contribution will be an amount not smaller than 0 ECU and not greater than 100 ECU. Furthermore, it must be an integer number. You will keep for yourself any amount of your endowment that you decide not to invest in the common project ("ECU you keep").

In every period, your earnings consist of two parts:

1. the "ECU you keep" = $[100$ - your contribution $]$ ECU;

2. the "income from the project".

The "income from the project" is calculated by adding up the contributions of the 3 members of your group and multiplying the resulting sum by a number that we call $\alpha$. That is:

Income from the project $=$ [Your contribution + Your partners' contribution $] \times \alpha$

The multiplier $\alpha$ is equal to 0.6. [Treatment HR] The multiplier $\alpha$ can be either 0.9 or 0.6 or 0.3 , [Treatment $L R]$ : The multiplier $\alpha$ can be either 0.75 or 0.6 or 0.45 , where each value is equally likely. You have to decide about your contribution without knowing the value of $\alpha$.

The income from the common project is determined in the same way for every member of the group, and it is independent of the size of your individual contributions.

[Treatments $H R$ and $L R]$ The 'income from the project' is determined as follows: each member is randomly assigned a number ranged between 1 and 3 regardless of the size of his/her contribution. Given that several (or all of them) members of the group can be assigned the same number, this is not necessarily a ranking. In case the extracted number is different for each subject, the subject with number 1 receives $\alpha=0.9$ [Treatment LR: $\alpha$ $=0.75]$ from the common project; the subject with number 2 receives $\alpha=0.6$, and the subject with number 3 receives $\alpha=0.3$ [Treatment $L R: \alpha=0.45$ ]. If it is the case that several (or even all subjects) subjects get the same number, ties are solved as follows:

- If all three members get the same number $(1,2$ or 3$)$, each member receives $\alpha=(0.9+0.6+0.3) / 3=0.6$ [Treatment LR: $\alpha=(0.75+0.6+0.45) / 3=0.6] ;$

- If two members get number 1 or 2 , they both receive $\alpha=(0.9+0.6) / 2=0.75$ [Treatment LR: $\alpha=(0.75+$ $0.6) / 2=0.675$ ] and the third member receives $\alpha=0.3$ [Treatment LR: $\alpha=0.45$ ];

- If two members get number 2 or 3 , they both receive $\alpha=(0.6+0.3) / 2=0.45$ [Treatment LR: $\alpha=(0.6+$ $0.45) / 2$ ] and the first ranked member receives $\alpha=0.9$ [Treatment $L R: \alpha=0.75$;

[Treatment BL] Example: If the sum of the contributions of the three members is $60 \mathrm{ECU}$, each member receives an income from the project equal to $(0.6 \times 60)=36 \mathrm{ECU}$.

[Treatment $H R]$ Example: If the sum of the contributions of the three members is $60 \mathrm{ECU}$ and each member is assigned a different number, the subject with number 1 receives an income from the project of $(0.9 \times 60)=$ 54 ECU [Treatment LR: $(0.75 \times 60)=45$ ECU]; the one with number 2 receives $(0.6 \times 60)=36$ ECU and the subject with number 3 receives $(0.3 \times 60)=18 \mathrm{ECU}$ [Treatment $L R:(0.45 \times 60)=27 \mathrm{ECU}$ ]. However, for instance:

- if all members are assigned the same number $(1,2$ or 3$)$ each receives $(0.6 \times 60)=36$ ECU;

- if two members are assigned the same number $(1$ or 2$)$, they each receive $[(0.9+0.6) / 2 \times 60]=45$ ECU [Treatment LR: $[(0.75+0.6) / 2 \times 60]=0.67 \times 60=40.2 \mathrm{ECU}]$; the other subject receives $(0.3 \times 60)=18$ ECU [Treatment LR: $(0.45 \times 60)=27$ ECU];

- if two members are assigned the same number $(2$ or 3$)$ they each receive $[(0.6+0.3) / 2 \times 60]=27 \mathrm{ECU}$ [Treatment LR: $[(0.6+0.45) / 2 \times 60=0.525 \times 60=31.5 \mathrm{ECU}]$; the other member of the group receives $(0.9$ $\mathrm{x} 60)=54 \mathrm{ECU}[$ Treatment LR: $(0.75 \times 60)=45 \mathrm{ECU}]$. 
At the end of each period you will receive information about the individual contribution of your partners in the group and your own period-earnings. Before the experiment starts, you will have to answer some control questions to verify your understanding of the rules of the experiment. Please remain seated quietly until the experiment starts. If you have any questions please raise your hand. 